\title{
The effect of adaptation on odor detection*
}

\author{
B. BERGLUND $†$ and U. BERGLUND, University of Stockholm, Stockholm, Sweden \\ T. ENGEN††, Brown University, Providence, Rhode Island 02912 \\ and \\ T. LINDVALL $+\dagger \dagger$, Karolinska Institute, Stockholm, Sweden
}

The sensitivity of human Ss to a weak odor (hydrogen sulphide) following prior exposure to it varying in both duration $(0-5 \mathrm{~min})$ and concentration $\left(4.05 \times 10^{-7}\right.$ to $24.40 \times 10^{-7} \mathrm{mg} /$ liter) was studied with a yes-no detection method and a specially designed olfactometer. The effect of adaptation was demonstrated, because the proportion of hits was lower the higher the concentration of the adaptation stimulus, when the response criterion, as measured by the proportion of false alarms, was fairly constant. By contrast, the proportion of hits was apparently independent of the duration of the exposure to the adaptation concentration. This finding suggests that the sense of smell is more stable than is usually believed. The paper ends with a discussion of the importance of considering adaptation in contemporary detection theory.

This is a report of experiments on the effect of intensity and duration of adaptation on odor detection (hit rate) and response bias (false alarm). The scanty literature on this topic shows that a S's sensitivity to an odorant will be less the stronger the prior adaptation to that odorant. However, this literature provides at least two different descriptions of this change in sensitivity as a function of duration of exposure. In Zwaardemaker's experiment of 1895 , which is most often quoted, it was found that as measured by absolute threshold, olfactory sensitivity decreased linearly during constant stimulation (cf. Pfaffmann, 1951). Recently, Ekman, Berglund, Berglund, and Lindvall (1967), who observed the effect of adaptation to suprathreshold concentrations, found that although perceived intensity of hydrogen sulphide decreased rapidly at first, this decrement then becomes more gradual in an exponential function of duration of exposure. It is often claimed that a person who enters a room containing a noticeable or even strong odor will soon become unaware of the odor. Evidence for this common notion of complete disappearance

* This investigation was supported by the Swedish Environment Protection Board, the Swedish Medical Research Council, and the Folksam Research Board. The study was also supported by research grants to Dr. Gösta Ekman from the Swedish Council for Social Science Research and the Bank of Sweden Tercentenary Fund. The authors are indebted to Mr. Gunnar Rosén and Mr. Per-Olof Olsson for help in performing the experiment.

+Address: Psychological Laboratories, University of Stockholm, Box 6801, S-11386 Stockholm, Sweden.

t† Address: Walter S. Hunter Laboratory of Psychology, Brown University, Providence, Rhode Island 02912.

$\dagger \dagger \dagger$ Address: Department of Environmental Hygiene, The Kurolinska Institute, S-10401 Stockhoim, Sweden. of the odor was obtained from only one of the eight Ss used by Ekman et al. For all the other $S s$, all concentrations (0.7-6.4 ppm) remained effective and at an appreciable and apparently constant level of subjective intensity and at no time presented a problem of detection.

In a scaling experiment, Cain and Engen (1970) showed that in olfaction, as in other sense modalities (J.C. Stevens \& S.S. Stevens, 1963), the effect of adaptation increases the slope of the psychophysical function. In other words, the weaker the stimulus (e.g., threshold concentration) the more it will be affected by prior adaptation. However, an interesting finding of that study was that regardless of the strength of the adaptation stimulus, its duration of presentation seemed to have no effect. That is, the effect of the adaptation concentration on the slope of the psychophysical function was essentially the same for $3,5,8$, and 15 inhalations. These data and those obtained by Ekman et al are contrary to the notion that odor will disappear with constant exposure, but seen to be consistent with recent neurophysiological evidence that the olfactory system of various animal preparations respond with good consistency to repeated stimulation (Ottoson, 1956).

There is one shortcoming of all the psychophysical experiments on the effect of duration of adaptation which may explain the discrepancies in the function relating odor sensitivity to duration of exposure. It is a well-known fact that a S's expectation of a sensory event will bias him toward "perceiving" it. For example, Woodrow and Karpman (1917) instructed their Ss to report when the odor of a constant stimulus disappeared, and by that instruction undoubtedly created the expectation that it would indeed disappear. Even if the $\mathrm{E}$ does not supply a bias, the $\mathrm{S}$ may develop his own conception of the expected outcome during the course of the experiment and tend to respond accordingly. In general, the instructions used in psychophysical experiments discourage biased responding, but it has been demonstrated that one must include in the experiment an independent index of the S's response bias in order to obtain a clear picture of sensitivity per se (Green \& Swets, 1966). The main purpose of the present experiment was to study the effect of adaptation on odor sensitivity with a detection method and to include a measure of response bias.

\section{THE EXPERIMENT}

\section{Subjects}

Individual data were obtained from two 20-year-old women (MR and CR), who were psychology students without any prior experience in psychophysics. They were paid SKr 10 (ca. \$2) per hour and received extra money for their performance according to a payoff matrix.

\section{Equipment and Materials}

The experiment was performed in a mobile olfactory laboratory unit consisting of two house trailers (Lindvall, 1970). The air in this laboratory, including the waiting room, the apparatus room, and the isolated test booth, was conditioned and purified with active carbon filters and absolute filters at a high flow rate $\left(550 \mathrm{~m}^{3} / \mathrm{h}\right)$. The temperature and humidity were maintained at $21^{\circ} \pm 2^{\circ} \mathrm{C}$ and $45 \% \pm 10 \%$ relative humidity, respectively.

The odorants were presented in two exposure hoods (A), one for the test stimulus and one for the adaptation stimulus, placed side by side in the test booth, as shown in Fig. 1. The concentration of the odorant, hydrogen sulphide, was kept in a cylinder (B) and was added in specified doses to a constant flow of purified nitrogen in a mixing unit (E) by the use of a capillary-manometer (C). The hydrogen sulphide was maintained at a constant pressure by a simple manostate (D). The nitrogen was kept in cylinder (F) and led through an active-carbon filter $(G)$ and a flow meter (H) to a mixing unit, from which superfluous gas could be regulated and evacuated by a valve.

The pressure of the mixture of nitrogen and hydrogen sulphide was controlled by a manometer ( $\mathrm{l}$ ) and a manostate ( $\mathrm{J})$ and led into an airstream $(\mathrm{K})$ through a glass tube (L) and steel capillaries (M). The purified air for this stream was flowed at a constant rate of $100 \mathrm{liters} / \mathrm{min}$. The mixture of purified air, nitrogen, and hydrogen sulphide was led into the exposure hoods and evacuated at such a rate that there was 


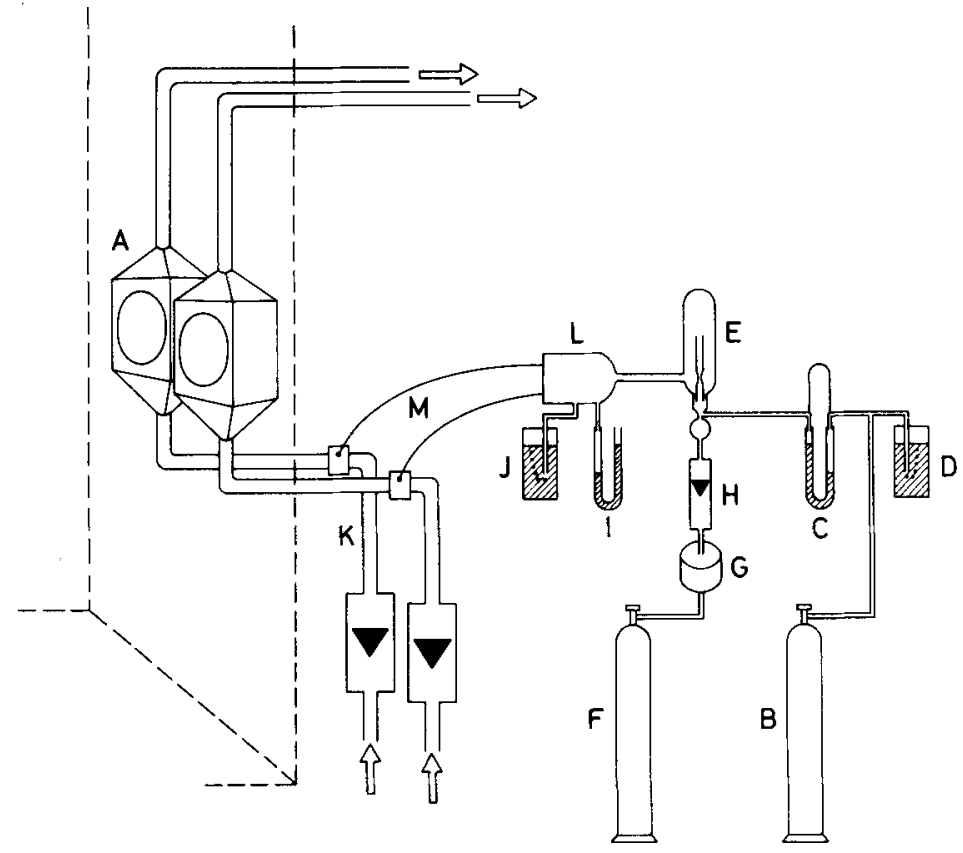

Fig. 1. The olfactometer used for control of concentration and stimulus presentation. (See text for details.)

a small negative pressure in the hoods in order to avoid leakage of the odorant into the isolated test booth. The oval openings of the hoods were just large enough to accommodate the S's face, and a tight fit was obtained by lining the sides of the openings with odorless silicone rubber. The capillaries $(M)$ feeding into the hoods were controlled with electromagnets, and it was possible to change the concentration of the odorants in $15 \mathrm{sec}$, including the time required to reach equilibrium. A battery of such capillaries and electromagnets provided a wide range of concentrations in geometric steps from which the test and the adaptation stimuli were selected.

The concentration of these gases was evaluated by a gas chromatograph. The hydrogen sulphide in nitrogen was sampled from the glass tube (L) and analyzed three times during each session with an electron capture detector in an Aerograph (204 B), using a Porapak Q $\left(75 \mathrm{~cm}, 75^{\circ} \mathrm{C}\right)$ column and nitrogen as the carrier gas. The gas chromatograph was calibrated at least twice daily against a mixture of hydrogen sulphide in nitrogen, which was kept at a constant temperature in a mylar plastic bag and analyzed according to a wet chemical standard method (Jacobs, Braverman, \& Hochheiser, 1957). The concentration in the glass tube was $9.70 \times 10^{-2} \mathrm{mg} /$ liter, with a relative standard error of $1.4 \%$ based on 37 observations. The capillaries were calibrated weekly and showed essentially constant flow rate.

\section{Stimuli}

Hydrogen sulphide (Matheson, Tech.) diluted in nitrogen was used both as the test and as the adaptation stimulus. $A$ concentration of $7.37 \times 10^{-7} \mathrm{mg} /$ liter was used throughout as the test stimulus. This was near the "absolute threshold" of both MR $\left(7.18 \times 10^{-7} \mathrm{mg} /\right.$ liter $)$ and CR $\left(5.14 \times 10^{-7} \mathrm{mg} / \mathrm{liter}\right)$ as determined by preliminary experiments with a modified method of limits (Lindvall, 1970). The two adaptation stimuli were $0.26 \mathrm{log}$ units above and $0.26 \mathrm{log}$ units below the concentration of the test stimulus. Purified air was presented instead of the test stimulus on the noise trials.

\section{Experimental Procedure}

Following extensive preliminary trials with the Es used as Ss, the whole experimental procedure was programmed on magnetic tape for automatic presentation of both the auditory and experimental session proceeded as follows: A short $(0.5-\mathrm{sec})$ tone from a loudspeaker signaled the $S$ to place her face in the test hood and to judge whether or not there was an odor in it by pressing the appropriate button on a panel at her fingertips. Then she was to exhale, move immediately over to the adaptation hood before the cessation of a second auditory signal, place her face in it, and breathe normally. The duration of the second signal was $2 \mathrm{sec}$ and the $S$ thus had altogether $4 \mathrm{sec}$ in which to make a judgment about the air in the test hood. After $16 \mathrm{sec}$ in the adaptation hood there was again a short tone telling her to repeat the same procedure as above. This visual signals and the stimuli. Each continued for $7 \mathrm{~min}$ for each experimental session, and each $\mathrm{S}$ participated in up to 30 sessions per day with at least 1 min rest between sessions. The durations of adaptation, recovery, and rest were determined on the basis of the research referred to above as well as preliminary experiments. There was a total of 180 sessions run per $S$ over a period of 2 months.

One session consisted of 21 periods in the adaptation hood and 22 judgments of the air sampled from the test hood. An olfactory stimulus was presented in the adaptation hood during the first $5 \mathrm{~min}$ or for the first 15 adaptation periods. The last 2 min of six periods in the adaptation hood were intended to test recovery and pure air was, therefore, substituted for the concentration of hydrogen sulphide in the adaptation hood. For each adaptation stimulus a total of 90 judgments were made by each $S$ for each of the 22 test points. The test stimulus was presented on half of these 90 trials and pure air on the other half. The order of presentation of test stimulus and pure air was randomized over groups of 10 sessions for each time point, and the adaptation stimuli were randomized between sessions. The Ss were not informed about any of the experimental conditions except the a priori probabilities of a test stimulus and pure air.

A symmetric payoff matrix was used to inform the $S$ about the accuracy of her performance by means of light signals mounted on the panel next to the response keys. She earned 10 Swedish öre (ca. 2 cents) for each correct judgment and lost 10 öre for each incorrect judgment. That is, during the moment of exhalation of the sample from the test hood the $S$ pressed a button to indicate "yes" or "no," and she was then immediately informed by a green or red light whether or not her response was correct. From the payoff matrix it was possible for the $S$ approximately to double her hourly pay.

\section{RESULTS}

The data for both Ss are presented in Table 1. The proportions of false alarms and hits are presented for both the adaptation and the recovery period. It is evident that CR tends to have a higher proportion of hits and almost invariably a lower proportion of false alarms than MR. One would conclude from this that the difference in classical thresholds obtained in the preliminary experiments reflect a response bias rather than sensitivity. Computation of $\mathrm{d}^{\prime}$ would indicate that $\mathrm{CR}$ is more sensitive than MR. Except when noted, the results are otherwise similar for both $S$ s with respect to all the points of interest in the present experiment.

Inspection of Table 1 indicates neither 
Table 1

The Proportion of Hits and False Alarms (FA) for Two Adaptation Intensities, $4.05 \times 10^{-7}$ (I) and $13.40 \times 10^{-7}$ (II) $\mathrm{mg} / 1$. (Each proportion is based on 45 judgments.)

\begin{tabular}{|c|c|c|c|c|c|c|c|c|}
\hline \multirow{3}{*}{$\begin{array}{l}\text { Dura- } \\
\text { tion in } \\
\text { Seconds }\end{array}$} & \multicolumn{4}{|c|}{ MR } & \multicolumn{4}{|c|}{ CR } \\
\hline & \multicolumn{2}{|c|}{1} & \multicolumn{2}{|c|}{ II } & \multicolumn{2}{|c|}{ I } & \multicolumn{2}{|c|}{ II } \\
\hline & Hit & $\mathbf{F A}$ & Hit & $\mathbf{F A}$ & $\mathrm{Hit}$ & $\mathrm{IAA}$ & Hit & $\mathrm{I} A$ \\
\hline 0 & .71 & .31 & .71 & .29 & .87 & .42 & .82 & .22 \\
\hline 20 & .82 & .49 & .60 & .58 & .58 & .07 & .56 & .06 \\
\hline 40 & .87 & .58 & .60 & .42 & .69 & .13 & .54 & .14 \\
\hline 60 & .71 & .33 & .53 & .40 & .71 & .11 & .62 & .20 \\
\hline 80 & .56 & .31 & .58 & .24 & .69 & .11 & .60 & .12 \\
\hline 100 & .76 & .27 & .49 & .27 & .84 & .13 & .60 & .18 \\
\hline 120 & .69 & .22 & .51 & .20 & .80 & .11 & .60 & .14 \\
\hline 140 & .60 & .33 & .56 & .33 & .67 & .07 & .70 & .00 \\
\hline 160 & .64 & .20 & .53 & .27 & .69 & .11 & .60 & .10 \\
\hline 180 & .58 & .27 & .62 & .33 & .76 & .11 & .70 & .04 \\
\hline 200 & .56 & .31 & .51 & .22 & .71 & .09 & .54 & .22 \\
\hline 220 & .64 & .36 & .51 & .29 & .80 & .13 & .66 & .08 \\
\hline 240 & .62 & .31 & .64 & .24 & .87 & .16 & .48 & .12 \\
\hline 260 & .73 & .29 & .53 & .36 & .78 & .11 & .48 & .08 \\
\hline 280 & .62 & .31 & .58 & .31 & .67 & .20 & .68 & .06 \\
\hline 300 & .73 & .38 & .62 & .36 & .71 & .04 & .58 & .16 \\
\hline \multicolumn{9}{|c|}{ Purified Air } \\
\hline 320 & .67 & .44 & .51 & .42 & .67 & .07 & .76 & .12 \\
\hline 340 & .73 & .24 & .65 & .25 & .78 & .07 & .82 & .12 \\
\hline 360 & .76 & .36 & .65 & .38 & .78 & .04 & .70 & .14 \\
\hline 380 & .73 & .31 & .80 & .35 & .78 & .04 & .70 & .10 \\
\hline 400 & .73 & .22 & .75 & .28 & .82 & .04 & .68 & .12 \\
\hline 420 & .77 & .24 & .72 & .33 & .68 & .16 & .68 & .12 \\
\hline
\end{tabular}

an increment nor a decrement of the proportion of hits as a function of duration of the period devoted to exposure to either adaptation stimulus. Likewise, there is no obvious change in the proportion of hits during the period of recovery. The proportion of false alarms is relatively constant and indicates that the response criterion was stable. For the present purpose one may utilize the proportion of hits as an index of odor sensitivity for each individual separately. Since there is no apparent effect of duration, the data may be averaged over time, and these results are presented in Table 2 .

The average proportion of hits for the recovery period is higher and the average proportion of false alarms is lower than those for the adaptation period. This indicates that the judgmental task was easier during the recovery period. It may be noteworthy that in the case of the weakest adaptation stimulus $\left(4.05 \times 10^{-7} \mathrm{mg} /\right.$ liter $)$ for CR the increase in the proportion of hits is relatively small and the decrease in false alarms relatively large.

Table 2

Averaged Proportion of Hits and False Alarms (FA) for Two Adaptation Intersities, $4.05 \times 10^{-7}(1)$ and $13.40 \times 10^{-7}($ II $) \mathrm{mg} / 1$

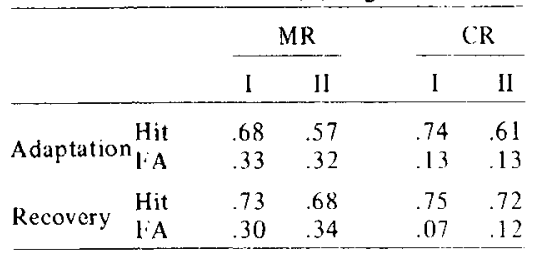

Without exception, the effect of the adaptation intensity is in the expected direction; that is, the stronger the adaptation stimulus, the lower the proportion of hits. At the same time, there is no discernable effect on the false alarms associated with adaptation intensity, and it is evident that this reflects a change in sensitivity rather than in the response criterion.

Near the completion of this experiment, CR became available for further experimentation. It was decided that most effective use of this time would be to test a stronger adaptation stimulus, the most effective variable observed in the present experiment. The concentration of this adaptation stimulus was $0.52 \log$ units above the test stimulus, and it was presented by itself in an experiment otherwise identical to the original. The results of this experiment are presented in Table 3.

It is clear that this adaptation stimulus is associated with the lowest proportion of hits and substantiates the finding that this proportion depends on stimulus intensity. It should also be noted that there is again apparently no change in the proportion of hits over time, but there is an indication that the proportion of false alarms is higher, especially at the onset of adaptation. If the proportion of hits were corrected by the proportion of false alarms, for example by computing $d^{\prime}$, it would indicate the unusual finding that sensitivity increases during the course of adaptation.
Finally, there is in this case no evidence of recovery, for the mean proportion of hits is 0.48 during adaptation compared with a mean of 0.50 during recovery. The mean proportions of false alarms are 0.19 and 0.17 for adaptation and recovery, respectively. Comparison of all the adaptation stimuli suggests that recovery from adaptation is slow compared with the temporal effect of adaptation on sensitivity. Tables 1 and 3 indicate that the effect of duration of adaptation is probably established between the first trial (unadapted at $0 \mathrm{sec}$ ) and the second, after $20 \mathrm{sec}$ of adaptation.

\section{DISCUSSION}

\section{Adaptation Intensity and Response Bias}

In agreement with both classical and contemporary psychophysical data referred to above, the effect of adaptation is proportional to the intensity of the adaptation stimulus. In this case, the stronger the stimulus the person is exposed to previously, the less likely he is to detect another weak stimulus of the same quality.

This conclusion is reinforced by the finding that the response criterion was relatively constant for different adaptation intensities. One exception to this was suggested for the strongest adaptation intensity, which was also tested separately. The possibility remains that different experimental conditions may produce different response biases. For example, Woodrow and Karpman's (1917) instructions to report when the odor disappeared may have increased the

Table 3

The Proportion of Hits and False Alarms (FA) Obtained for an Adaptation Stimulus of $24.40 \times 10^{-7}$ (III) $\mathrm{mg} / 1$

\begin{tabular}{|c|c|c|}
\hline \multirow{3}{*}{$\begin{array}{l}\text { Duration } \\
\text { in Seconds }\end{array}$} & \multicolumn{2}{|c|}{ CR } \\
\hline & \multicolumn{2}{|c|}{ III } \\
\hline & Hit & $1 \because \mathrm{A}$ \\
\hline 0 & .40 & .30 \\
\hline 20 & .35 & .15 \\
\hline 40 & .50 & .35 \\
\hline 60 & .50 & .25 \\
\hline 80 & .40 & .13 \\
\hline 100 & .45 & .25 \\
\hline 120 & .55 & .23 \\
\hline 140 & .45 & .18 \\
\hline 160 & .53 & .15 \\
\hline 180 & .38 & .28 \\
\hline 200 & .43 & .20 \\
\hline 220 & .48 & .15 \\
\hline 240 & .55 & .08 \\
\hline 260 & .53 & .05 \\
\hline 280 & .55 & .13 \\
\hline 300 & .43 & .13 \\
\hline & Air & \\
\hline 320 & .35 & .20 \\
\hline 340 & .58 & .18 \\
\hline 360 & .43 & .18 \\
\hline 380 & .48 & .15 \\
\hline 400 & .63 & .13 \\
\hline 420 & .50 & .18 \\
\hline
\end{tabular}


likelihood of a "miss," while the request of Ekman et al (1967) for a judgment of odor intensity may suggest it will not disappear and thus result in a tendency toward "false alarms." In the present case, increasing the adaptation stimulus increases the difficulty of the task and reduces the $S$ 's earnings with as yet unknown psychological consequences. The observation of response bias was an important aspect of the present experiment, and it is surely one of the important and yet largely unexplored problems of sensory psychology.

\section{The Effect of Exposure Duration}

Although the intensity of adaptation affects the S's sensitivity, it is evidently not the case that it will disappear. While $\mathrm{Zwaardemaker}$ found that sensitivity decreased monotonically with duration of exposure as measured by threshold, the present experiment suggests that sensitivity is very stable and unaffected by duration. It is as though the state of adaptation due to the intensity of the adaptation stimulus sets the limit for the sensitivity of the system and thus determines sensitivity, as in a signal-to-noise model. This finding is consistent with the evidence from recent experiments in psychophysical scaling of relatively intense stimuli (Cain \& Engen, 1970; Ekman et al, 1967).

\section{Rate of Adaptation and Recovery}

There remains one important puzzle requiring fusther experimentation. The data by Ekman et al (1967), for example, show that the sensitivity of the Ss does not go to zero or fall below threshold, but in their case there was a marked decrease in the perceived intensity of the suprathreshold stimuli during the course of the first $300 \mathrm{sec}$, at which time the judgments reached a stable level. In the present case, with weak stimuli, the effect of adaptation appears to be much more rapid and may even be an immediate result of the onset of stimulation. By comparison, recovery from adaptation appears to be slower than the adaptation process in the present data as well as in those by Ekman et al (1967). This problem is of great potential interest for sensory psychology, and it is unfortunate that the present data are not sufficiently reliable to provide a nonadapted reference point of detectability. Likewise, the data suggest the possibility that recovery from adaptation may be indicated by either a higher proportion of hits or a lower proportion of false alarms. In any case, the present experiment illustrates the value of detection methodology, and further application of detection theory will undoubtedly lead to improved understanding of sensory adaptation.

\section{Adaptation and Signal Detection Theory}

In determining the sensitivity of a sensory system by requiring a $S$ to detect a signal in noise, two types of functional relationships are particularly informative. One is the psychometric function relating the percentage of correct detections (hits) to stimulus intensity, and the other is the receiver operating characteristic (ROC), or isosensitivity function. The present investigation has dealt with the former relation, although in a novel context, by studying the proportion of hits for only one stimulus as a function of the adaptation state. Before applying any of the different models of signal detection theory to adaptation data, the probability density functions of noise and signal must be considered with reference to the psychological aspects of the experimental data. The ROC curve then becomes of considerable potential value.

In the present experiment the use of a Gaussian distribution model with equal variances would entail the possible assumption, for example, that the signal distribution remains relatively fixed but that the noise distribution is moved along the excitation axis due to adaptation. From a physiological point of view it is not possible to consider the noise distribution as in an ordinary signal detection experiment, because the adaptation stimulus must affect the events associated with both stimulus and noise. Therefore, it seems more reasonable to assume (1) that the variances will become unequal due to adaptation, or (2) that both distributions vary along the excitation axis in the same direction as a result of duration of adaptation. The latter would yield an approximately constant $d^{\prime}$, and in that sense it is possible that an adaptive effect did indeed result from duration in the present experiment

With sufficient data to determine the form of the ROC curve for each observation point along the adaptation function, it would be possible to test the former assumption (1) regarding a Gaussian distribution with unequal variances. If the
ROC curve becomes "asymmetric" due to adaptation, the introduction of an exponential distribution (cf. Green \& Swets, 1966, p. 78.81) would be preferred since it also implies a monotonic relationship between the likelihood ratio and the detection axis. In fact, this model has been applied and proved to be useful in a study of uncertainty as a function of the occurrence of an auditory signal in time (Egan, Greenberg, \& Schulman, 1961). Given further refinements in the mathematical formulations, the effect of adaptation can be handled by the exponential distribution model.

It seems likely that consideration of sensory processes, like adaptation, can and should be made for the potential theoretical contribution this might provide in return for a more general and applicable theory of detection.

\section{REFERENCES}

CAIN, W. S, \& ENGEN, T. Olfactory adaptation and the scaling of odor intensity. In $C$, Pfaffmann (Ed.), Olfaction and taste. New York: Rockefeller University Press, 1969. Pp. 127-141.

EGAN, J. P., GREENBERG, G. Z., \& SCHULMAN, A. I. Interval of the time uncertainty in auditory detection. Journal of the Acoustical Society of America, 1961, 33, 771-778.

EKMAN, G., BERGLUND, B., BERGLUND, U., \& LINDVALL, T. Perceived intensity of odor as a function of time of adaptation. Scandinavian Journal of Psychology, 1967, 8, 177-186.

GREEN, D. M., \& SWETS, J. A. Signal derection theory and psychophysics. New York: Wilcy. i966.

JACOBS, M. B., BRAVERMAN, M. M. \& HOCHHEISER, S. Ultramicrodetermination of sulphides in air. Analytical Chemistry, 1957. 29, 1349-1351.

LINDVALL, T. On sensory evaluation of odorous air pollutant intensities, Nordisk Hygienisk Tidskrift, 1970, Supplement 2, $1-181$.

OTTOSON, D. Analysis of the electrical activity of the olfactory epithelium. Acta Physiologica Scandinavica, 1956, 35, Supplement 122. 1-83.

PFAFTMANN, C. Taste and smeli. In S. S. Stevens (Ed.), Handbook of experimental psychology. New York: Wiley, 1951.

STEVENS, J, C., \& STEVENS, S. S. Brightness function: Effects of adaptation. Journat of the Optical Society of Amcrica, 1963, 53, $375 \cdot 385$.

WOODROW, H. I, \& KARPMAN, B. A new olfactometric technique and some results. Journal of Experimental Psychology, 1917, 2, $431-447$.

(Accepted for publication November 1. 1970.) 